\title{
Flexible FPGA-Based Controller Architecture for Five-fingered Dexterous Robot Hand with Effective Impedance Control
}

\author{
Z.P. Chen, N.Y. Lii, K. Wu, H. Liu \\ Institute of Robotics and Mechatronics, \\ German Aerospace Center, DLR \\ 82234 Wessling, Germany \\ zhaopeng.chen@dlr.de
}

\author{
Z.X. Xue \\ Forschungszentrum Informatik (FZI) \\ Haid-und-Neu-Str. 10-14 \\ 76137 Karlsruhe, Germany \\ xue@fzi.de
}

\author{
M.H. Jin, Y.W. Liu, S.W. Fan, T. Lan \\ State Key Laboratory \\ of Robotics and System \\ Harbin Institute of Technology \\ 150001 Harbin China
}

\begin{abstract}
Several practical issues associated with achieving effective impedance performance in the finger joint space and stable grasping on a five-fingered dexterous hand are investigated in this work. A Multiprocessor structure based on field programming gate array (FPGA) is proposed to realize the high-level hand impedance controller. The key feature of the hardware system is a dual-processor architecture based controller, one of which is used for data communication control and the other for joint and object level control. High speed (200 $\mu$ s cycle time) multipoint low-voltage differential signaling (LVDS) serial data bus communication between each finger and the controller, Ethernet communication between monitor PC and controller, are all implemented on a single FPGA chip. Experimental results and simulation with a five-fingered dexterous robot hand demonstrate that the controller architecture is able to achieve the desired robot hand impedance control performance and effective stable grasping.
\end{abstract}

Index Terms - dexterous hand, FPGA, flexible communication system, impedance control, grasping

\section{INTRODUCTION}

In order to improve the manipulative capability of a robot, the dexterous robot hand has become one of the most important research aspects in the robotics field. In recent years, several anthropomorphic robot hands have been developed, including the NASA Robonaut Hand [1], the Gifu III Hand [2], the Shadow Hand G5, the DLR Hand II [3] and the DLR/HIT Hand I [4]. Robot hands may be classified into two categories. First is the externally actuated hand, which utilizes actuators mounted in the forearm (NASA and Shadow). The second category employs internal actuation (DLR, HIT), with all actuators and electronics integrated in the finger and the palm [5]. Several features are in common between both categories of robot hands mentioned above: highly integrated modularity and dexterity design that involve mechanical and electronic architecture. The electronic architecture significantly influences the control and manipulation performance, as well as the reliability and stability of the hand. Furthermore, the electronic hardware affects the design integration and modularity of the mechanical architecture of the robot hand. With compact electronic design and advancement in several technologies, such as novel sensors, processors and actuators, smaller and more anthropomorphic robot hand can now be realized. Specifically designed communication protocols and electronic board-level connections are necessary to help fulfill modular design of the robot hand.

Control algorithm progress in the dexterous robot hand demands higher requirements including high-speed data transfer, fast calculation, dependable communication and more data/program memory, etc. Field programmable gate array (FPGA) has become a viable option in complex logic circuit design due to its flexibility, ease to use, and short time to market [6]. The FPGA is suitable for flexible and complex logical structures, including data processing and communication system. Processing speed can be further increased with multi-processor parallel processing architecture. Some researchers have implemented control algorithms into FPGA to improve the performance of the servo control system. Takahashi and Goetz developed a system to run a current control algorithm with the aid of an FPGA to increase the bandwidth of the current loop control [7]. Vector and velocity controls of a PMAC servo motor based on FPGA have also been successfully realized by Tzou and Kuo [8]. Another work concerning FPGA-based motion control could be found in [9].

Robot hand control focuses on performing compliant manipulation and reliable grasping with different objects in an unknown environment. Impedance control is one possible approach to achieve compliant behavior, which was extensively theorized by Hogan [10] and experimentally applied by Kazerooni [11]. The basic principle of the impedance law is defined as a mass-spring-damp system, where the interacting force is designed to be a linear combination of the position error (spring), first derivative of the position (damping), and second derivative of the position (inertia). The equations of a manipulator's motion are usually derived in terms of joint coordinates, which is called joint space. Finger joint space 
control performance are the fundamental features of the robot hand control.

This paper focuses on designing a novel hardware architecture to enhance a dexterous robot hand's impedance performance and achieve embedded intelligent hand grasping. This includes the following two aspects: (1) utilization of FPGAs to achieve ideal hardware architecture for impedance control and embedded intelligent hand grasping, and (2) flexible communication system to fulfill control requirements in different operating environments, supporting multipoint low-voltage differential signaling serial bus, Ethernet, and CAN bus. All design aspects are realized in the DLR-HIT II five-fingered dexterous robot hand.

This paper is organized as follows: Section II gives an overview of the hardware and software architecture of the FPGA-based control system; Section III describes the flexible communication system architecture; Section IV presents experiments and performed a grasping application. Finally, the conclusions of this work are drawn and presented in Section $\mathrm{V}$.

\section{Architecture of FPGA-BASEd CONTROLLER IN DLR-HIT HAND II}

The DLR-HIT-Hand II is a multisensory integrated fivefingered hand with a total of 15 DOFs (degrees of freedom), as shown in Fig. 1. All actuators, electronic components, and communication controllers for a finger are fully integrated in the finger base of the finger body. To be more anthropomorphic and capable of complex tasks, the controller must be small in size, and possess powerful data processing capability. In order to achieve the desired performance, the robot control system relies not only on the control algorithm, but also on the hardware and software architecture of the controller.



Fig. 1. 5-fingered dexterous robot hand DLR-HIT-hand II

\section{A. Hardware architecture}

As shown in Fig. 2, the FPGA provides the processing for the finger base joint motor control, which is physically located at the bottom of a finger base. A Cyclone II FPGA with 18752 logic elements and $234 \mathrm{Kbits}$ of RAM is chosen

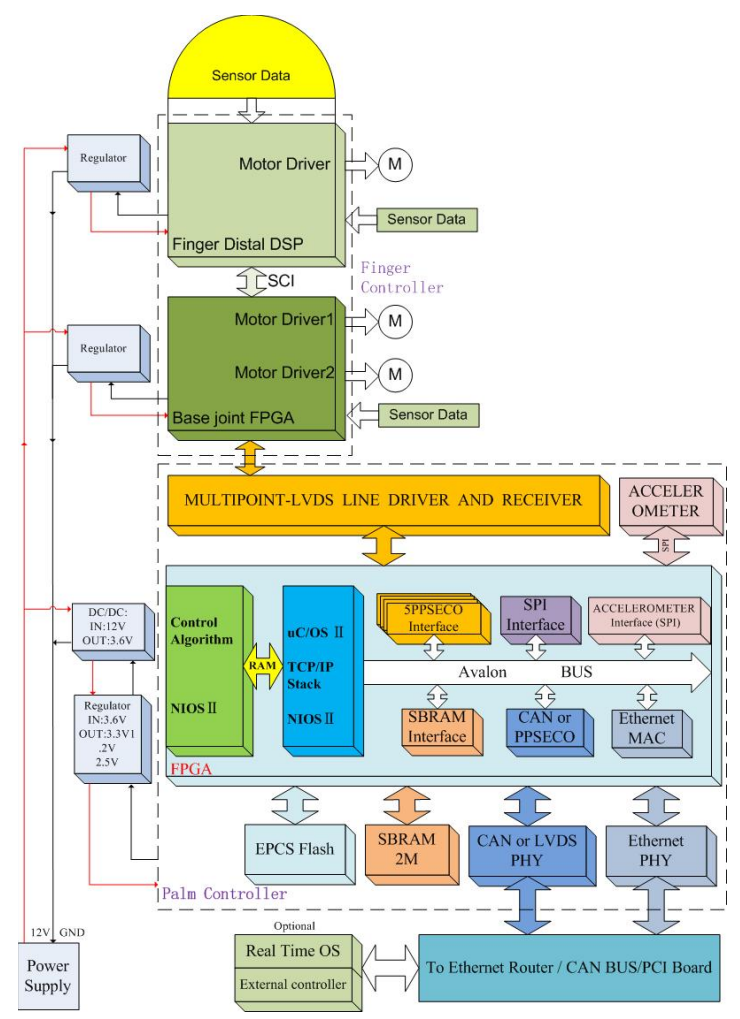

Fig. 2. Controller Diagram

for the this task. All the motor driver, communication with finger distal and higher control level, sensor data acquiring are implemented in one single electronic board with Very-High-Speed Integrated Circuit Hardware Description Language, (VHDL). Two brushless DC (BLDC) motors are directly driven and controlled by an FPGA with MOSFET driver gates. In order to achieve modularity of the robot finger, all the parameters related to the finger control are stored in the flash memory on the finger base board. The finger distal motor is controlled by a DSP, which is attached on the back side of the first linkage of the finger. For the finger distal motor, a Texas instruments (TI) floating-point digital signal processor (DSP) with maximum 100 MMACS is selected to carry out the joint level control algorithm, sensor signals processing, BLDC motor control, as well as the communication between the finger distal and the finger base. With the aid of the finger DSP, the number of wires between the finger base and the finger has been reduced from 18 (in DLR/HIT Hand I) to 5. Fewer wires leads to a more reliable system and improved communication quality.

For higher level tasks the a Cyclone III FPGA with NIOSII dual-processors, 24624 logic elements, and 594 Kbits of RAM is chosen. With its sufficient processing power, an enhanced controller with more flexible communication system can be implemented in a smaller electronics package. Two separate NIOSII processors are implemented in the Cyclone 
III, with $2 \mathrm{k}$ bits on-chip RAM for inter-processor communication. One of the processors is responsible for processing impedance control and grasp planning algorithm. Communication tasks are controlled by the second processor. To ensure that the two processors can work efficiently together without conflict, a hardware mutex is utilized to protect critical resources shared by these two processors. By means of the SOPC (system on programable chip) builder tools, all modules are integrated and fully implemented on a single chip. With the advantage of flexible FPGA structure and integrated processing units, the proposed control architecture is able to achieve high control performance with a smallsized control hardware electronics. Furthermore, diverse I/O standards support connections between different hardware components (e.g. FPGA, DSP, motor, sensors), which makes the controller more flexible for different applications.

\section{B. Control Software Architecture}

Along with the controller hardware architecture in the robot hand, a multi-level modular structure is also adopted in the hand's software architecture. As illustrated in Fig. 3, data processing in software is implemented in three major logic levels, Data Processing Level, Lower Control Level and Higher Control Level.

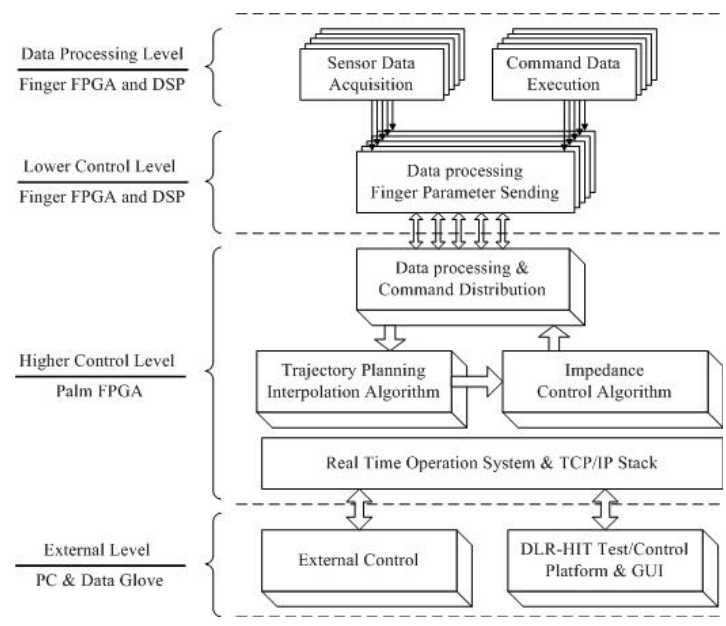

Fig. 3. Multilevel Structure of Control Software

All calculations required for the conversion of digitized sensor values to applicable measurements, such as position and torque, as well as derived values such as velocities, are fulfilled in the Data Processing Level. To eliminate disturbances and ripples in the motor hall signal and torque sensor values, a Butterworth filter is adopted to achieve a high resolution of velocity, position and torque measurements.

In the Lower Control Level, all data necessary for control algorithm are packed together and sent back to the Higher Control Level. Furthermore, basic motor drivers and control, system monitoring and safety tasks concerned with each finger joint sensor are also implemented in this control level. The control loops in all five fingers wait for the interrupt from the Higher Control Level, so that commands and measurements of all fingers are executed synchronously with the same hardware clock. Joint or Cartesian commands are then received from the Higher Controller Level, along with control parameters of different controllers, and the enable status of the motors. Since most of the parameters required for control algorithm are stored in the flash memory of the Lower Control Level, reading and sending these parameters back to the Higher Control Level is also processed in this level.

In the Higher Control Level, all algorithm run with a $200 \mu$ s cycle time to perform real time hand control. Data acquisition, unpacking and basic processing are the first steps of Higher Control Level. The next step is the receiving command values for the desired control parameters, such as position, velocity, and stiffness of each joint. The interpolation algorithm for trajectory planning can then process the received command values to interpolate control data. Since no smooth transition between the different types of controllers is provided in this control level, the user may choose from several controller for the task at hand. The implemented types of controllers include joint space impedance control, based on position with friction and gravity compensation, as well as a position controller. Finally, extensive safety checks are performed, including joint and temperature limits, sensor signal validity, and communication system integrity. The harmonic drivers in the finger distal, base, and special cardan base joints of DLR-HIT Hand II all require violation limit checks in this level, since workspace limitations have to be confirmed in true joint angles rather than the directly measurable motor or transmission angles.

\section{Flexible Communication System ARCHITECTURE}

A large amount of data has to be transmitted between joint level, Cartesian level and host level. The data package consists of motor control data, sensor data and tracking data. During communication, the joint level should pack all the digital sensor values, while Cartesian level should distribute the control signal to each finger, and send tracking data back to the host level at the same time. Furthermore, considering that robot hand might be used in different situations, such as industrial environment or with an external real-time controller, A more industry standard and universal interface between the hand and host level would be suitable. Table I compares some communication protocols in different robot hands.

\section{A. Between higher control level and lower control level}

To realize real-time feedback control of the robot hand, it is important that the higher control level receives the realtime positions and torques of the joints timely to calculate 
TABLE I

COMMUNICATION METHODS IN ROBOT HANDS

\begin{tabular}{lcc} 
Hands & $\begin{array}{c}\text { lower level } \\
\text { communication }\end{array}$ & $\begin{array}{c}\text { higher level } \\
\text { communication }\end{array}$ \\
\hline SDH Hand & RS232 & Ethernet, CAN \\
DLR Hand II & IEEE 1355 & IEEE, 1355 \\
GIfu hand III & none & PCI \\
Shadow hand C5 & none & Ethernet, CAN \\
DLR HIT Hand I & M-LVDS & M-LVDS \\
DLR-HIT Hand II & M-LVDS & Ethernet, CAN, M-LVDS
\end{tabular}

the velocities and quasi-steady state for the controller. On the other hand, the lower level should update the input control data instantly especially in fast-moving and complex situations. Therefore, a high speed data bus is needed between the lower level finger controller and the higher level hand controller in the proposed control architecture.

Multipoint low-voltage differential signaling (LVDS) allows multipoint configuration defined by the ANSI/TIA/EIA899 M-LVDS standard, which recommends a maximum data rate of $500 \mathrm{Mbps}$. Because of its low noise, low power consumption, high immunity interface, and ease of implementation in FPGA, by means of low-voltage differential line drivers and receivers, a M-LVDS serial bus system is designed for the data communication between the fingers and the palm controller, as shown in Fig. 4.

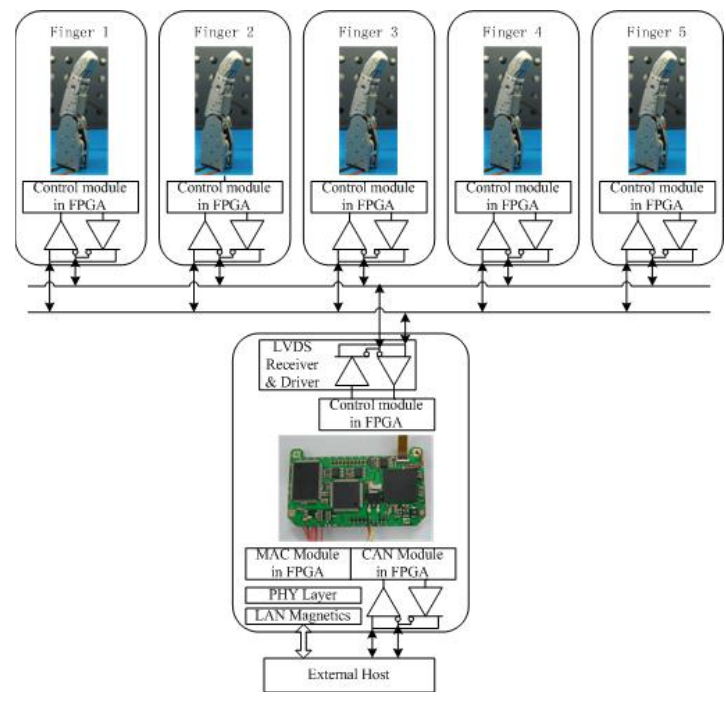

Fig. 4. M-LVDS serial data bus

The M-LVDS serial bus has the following characteristics :

1) LVDS Serial bus is composed of a Multipoint, halfduplex, and serial communication link, based on LVDS (Low-Voltage Differential Signaling) physical layer.

2) LVDS Serial bus uses a 16-bit cyclical redundancy check (CRC) checksum based on the CCITT poly- nomial to ensure that the majority of communication errors can be detected.

3) NRZI data encoding and automatic bit stuffing/stripping are used for data encoding.

4) The LVDS serial bus can accommodate variable baud rate. Unnecessarily high communication rate can reduce the control performance of whole system. In the proposed system a bandwidth of $25 \mathrm{Mbps}$ is chosen.

Based on the above specifications, the LVDS serial bus increases the transmission speed and reliability of data communication, reduces cabling and noise in signals. With VHDL and NIOS II soft processor the serial bus is implemented on FPGA and fully integrated into the five-fingered dexterous robot hand successfully.

\section{B. Between host level and controller level}

Communication data package between host and controller, consists of position, velocity, torque tracking signal and control data. To acquire the real-time feed back tracking signal and transfer manipulation control data, such as destination position, or required velocities, data should be transferred timely between host and controller. On the other hand, to make the hand more compatible for different applications, a universal communication protocol is necessary in the high level communication. For the above reasons, Ethernet and CAN were chosen to fulfill the requirements.

Ethernet is the most widespread wired local area network technology. To implement Ethernet in FPGA, Triple speed ethernet IP core from Altera has been chosen for the MAC layer of Ethernet. For the physical layer, DP83848T with RMII interface from TI has been selected. Along with MicroC/OS II real time operating system, NicheStack TCP/IP stack has also been ported into the system, which combines with the application program to realize $100 \mathrm{M}$ baud full duplex Ethernet network in the dexterous hand.

CAN (controller-area network) bus is based on an automotive communication standard designed to allow microcontrollers and devices to communicate with each other within a passenger vehicle without a host computer. It is designed specifically for automotive applications, but has since been employed for other applications. Considering the possible future usage in the industrial environment, CAN bus is supported in the dexterous hand. CAN controller IP core from IFI has been implemented, and SN65HVD230d from $\mathrm{TI}$ is chosen for the physical layer.

With the above communication methods, the dexterous robot hand is more flexible in control design and usage. Controllers could be implemented inside the hand, or realized by a real-time external controller with Ethernet or CAN. Grasp planing and manipulation strategies can be processed in the external controller so that more complex and meticulous manipulation could be accomplished by the five-fingered dexterous robot hand. Fig. 5 illustrates the transmission of 
control signal sequentially in $200 \mu$ s cycles, and receiving of all five fingers' feedback sensor data, while sending sensor tracking data and receiving commands from the host level in every $10 \mathrm{~ms}$.

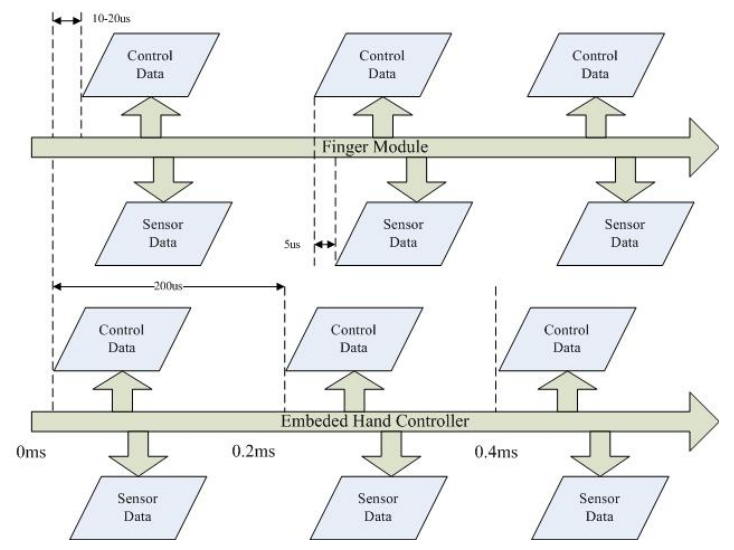

Fig. 5. Communication Architecture

\section{EXPERIMENTS AND GRASPING APPLICATION}

\section{A. Finger joint space impedance control}

Experiments are performed on the five-fingered dexterous robot hand. For each finger, two specially designed potentiometers in the base joint and a contactless magnetic angle sensor in the finger distal unit are equipped to measure the absolute angular position of the 3 joints. To precisely measure the external torque without hysteresis, a new type of base joint torque sensor with two DOFs and a distal torque sensor, both based on strain gauge theory, are adopted for the three joints.

The fingers are of modular design, with identical mechanical structure and control architecture. The principle of finger joint impedance control fulfills the requirement between external force and finger joint position by adjusting the stiffness parameters. The robot hand kinematics model [12] is well known, and shown below:

$$
\begin{gathered}
M(q)(\ddot{q})+C(q, \dot{q}) \dot{q}+g(q)=K(\theta-q)+\tau_{\text {ext }} \\
B \ddot{\theta}+K(\theta-q)+\tau_{F}=\tau_{m}
\end{gathered}
$$

where $M(q), C(q)$ and $g(q)$ represent the inertia matrices, centrifugal term, and gravity term, respectively.The vector of the joint torques is given by $K(\theta-q)$, where $\theta, q$ indicate the vector of the motor angle divided by the gear ratio and the link side joint angle. $K, B$ are diagonal matrices which contain the joint stiffness and the motor inertias multiplied by the gear ratio squared. The external torque vector, $\tau_{e x t}$, and the generalized actuator torque vector, $\tau_{m}$, are considered as the control inputs.

A classical position-based impedance controller (PBIC) [13] is realized to demonstrate the performance of the proposed hardware architecture. The block diagram of feedback control is shown in Fig. 6 where $\theta_{s r}$ and $\theta_{m r}$ are position sensor data from position sensors and motor hall sensors, respectively.

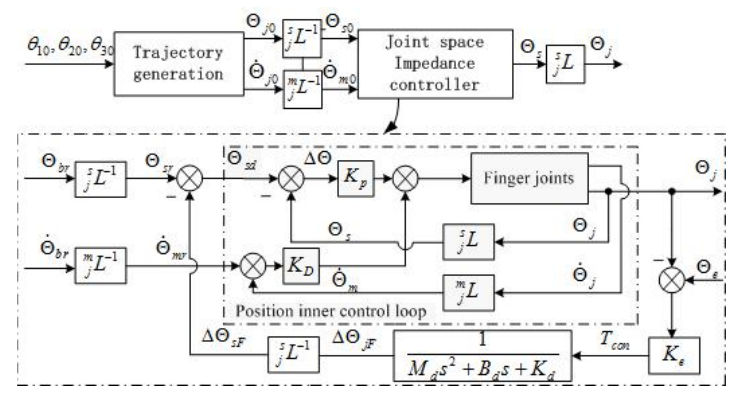

Fig. 6. Position-Based Impedance Control Architecture

In this experiment, each joint of the finger is independently driven. The aim is to make the finger move only upward and downward ( $x$ direction), coming in contact with an external object before reaching the desired position. Damping and stiffness are set at $D_{d}=[1.0,1.0,1.0]\left(\mathrm{N} \cdot \mathrm{mm} \cdot \mathrm{s} /{ }^{\circ}\right)$, $K_{d}=[0.15,0.5,0.5]\left(N \cdot m m /{ }^{\circ}\right)$. Fig 7 shows the finger base joint tracking the desired position trajectory (red line). In this example, contact is made with a rigid external object at a joint angle of $21^{\circ}$, where real tracking seperates from the desired tracking. The experimental results show that the joint can follow the desired trajectory closely in the free space, and the joint torque increases stably while it makes contact with the environment. It can therefore be concluded that the finger joint impedance behavior is successfully achieved.

\section{B. Intelligent hand grasping application}

The introduced five-fingered dexterous robot hand fulfills the requirement for grasping and manipulation. This is validated by grasping experiments performed with the robot hand. The kinematic and geometry models of the hand is imported into an automatic grasp planning system [14], based on the grasp simulator GraspIt! [15]. To plan stable grasps, the Cartesian frame between the robot hand and the object is approximated by grasping directions, which are generated from the geometric decomposition of the object model. The hand configuration space is reduced to a few predefined hand preshapes, torwards which the fingers close in the grasping simulation. Continuous collision detection technique is applied to locate the contact points between the robot hand and the object efficiently. From these contact points, the grasp wrench space is built. The stability of the grasp is computed in a 6-dimensional grasp wrench space. For stable grasps, the position matrix $\theta_{e}$ is computed with the given stiffness matrix $K_{d}=[0.15,0.5,0.5]\left(N \cdot \mathrm{mm} /{ }^{\circ}\right)$. The performed grasp is shown in Fig. 8.

\section{CONCLUSIONS AND FUTURE WORK}

A novel hardware controller architecture and flexible communication system with finger joint space impedance 

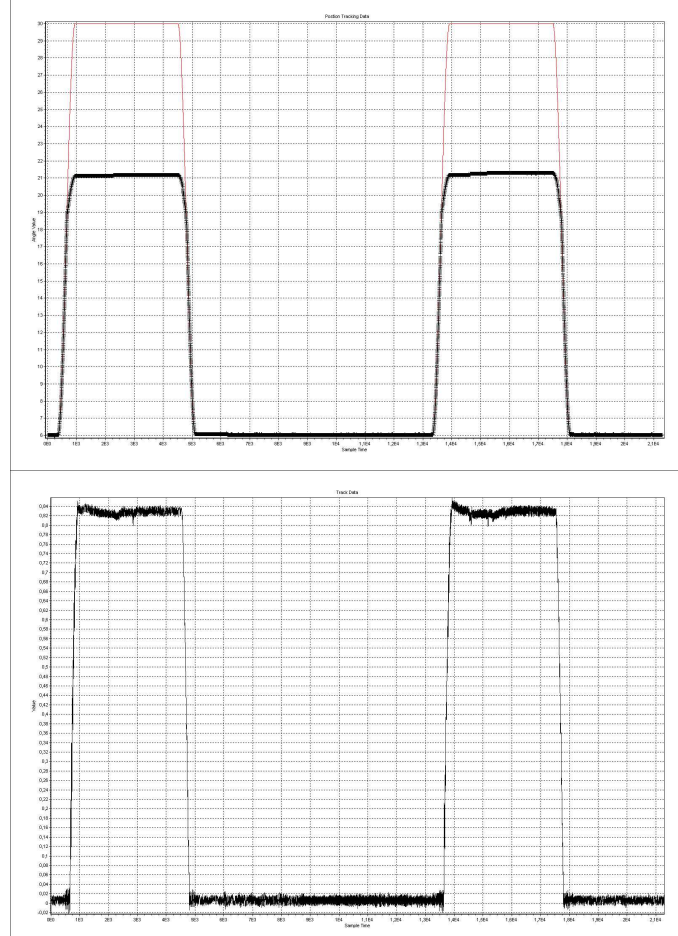

Fig. 7. Position and Force response tracking in $x$ direction
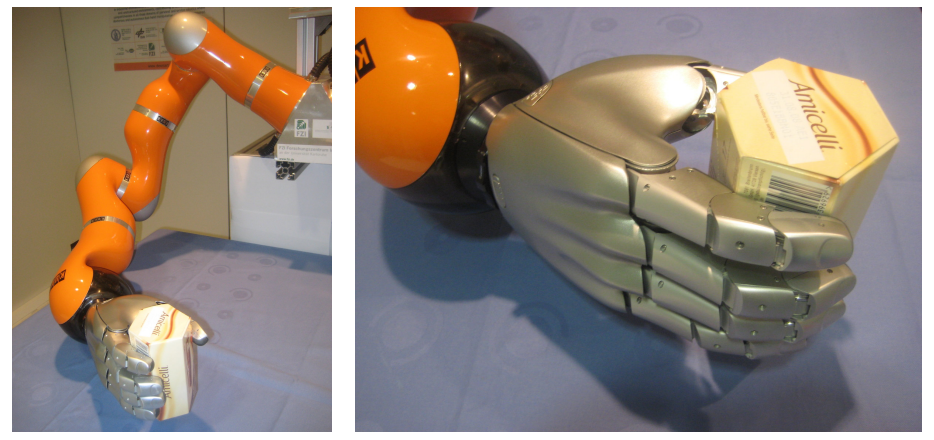

Fig. 8. Execution of automatically planned grasp

control is investigated in this paper, which is successfully implemented on the DLR-HIT II dexterous robot hand. The hardware and software system are designed to achieve sufficient data communication and control of the hand. All control and communication modules are implemented on a single FPGA chip with a dual-processor unit, M-LVDS serial bus with $200 \mu$ s cycle time, Ethernet with TIP/IP stack, and CAN bus. Integrated control hardware design concept has helped reduce the size of the robot hand by introducing more compact component level design. Experimental results, obtained on the five-fingered dexterous robot hand, verified that the FPGA-based control architecture have satisfactory behavior in impedance control and grasping.

In order to make the robot hand an intelligent device capable of guiding the robot arm to perform autonomous grasping, integration of the grasp planning system into the FPGA chip is currently underway. An adaptive torque based controller is currently being investigated to enable stable grasping of deformable or unknown objects.

\section{ACKNOWLEDGMENT}

The authors would like to thank Jiangbin Huang, Kui Sun at the Harbin Institute of Technology for the numerous research discussions. Deep gratitude also goes to $\mathrm{Mr}$. Peter Meusel, and Mr. Benedikt Pleintinger of the German Aerospace Center (DLR) for their technical support.

\section{REFERENCES}

[1] M. C.S.Lovchik, "The robonaut hand: A dextrous robotic hand for space," in Proceedings of the IEEE International Conference on Robotics and Automation, 1999, pp. 907-912.

[2] K. Y. Tetsuya Mouri, Haruhisa Kawasaki, "Anthropomorphic robot hand: Gifu hand iii international conference on control, automation and systems," in International Conference on Control, Automation and Systems, 2002, pp. 1288-1293.

[3] H. L. J. Butterfass, M. Grebenstein, "Dlr-hand ii: Next generation of a dexterous robot hand," in In Proceedings of the 2001 IEEE International conference on Robotics I\& Automation, 2001, pp. 109114.

[4] R. Wei, X. Gao, M. Jin, Y. Liu, H. Liu, N. Seitz, R. Gruber, and G. Hirzinger, "FPGA based hardware architecture for HIT/DLR hand," in 2005 IEEE/RSJ International Conference on Intelligent Robots and Systems, 2005.(IROS 2005), 2005, pp. 523-528.

[5] H. Liu, K. Wu, P. Meusel, N. Seitz, G. Hirzinger, M. Jin, Y. Liu, S. Fan, T. Lan, and Z. Chen, "Multisensory five-finger dexterous hand: The DLR/HIT Hand II," in IEEE/RSJ International Conference on Intelligent Robots and Systems, 2008. IROS 2008, 2008, pp. 36923697.

[6] J. Huang, Z. Xie, H. Liu, K. Sun, Y. Liu, and Z. Jiang, "DSP/FPGAbased Controller Architecture for Flexible Joint Robot with Enhanced Impedance Performance," Journal of Intelligent and Robotic Systems, vol. 53, no. 3, pp. 247-261, 2008.

[7] G. J. Takahashi T., "Implementation of complete ac servo control in a low cost fpga and subsequent assp conversion," in Appl. Power Electron. Conference Exposition 1, 2004, pp. 565-570.

[8] K. T. Tzou Y.Y., "Design and implementation of an fpga based motor control ic for permanent magnet ac servo motors," in Appl. Power Electron. Conference Exposition 1, 2004, pp. 565-570.

[9] e. Dubey R., Agarwal P., "Fpga based pmac motor control for systemon-chip applications," in Proceedings of First International Conference on Power Electronics Systems and Application, 2004, pp. 194-200.

[10] N. Hogan, "Impedance control-An approach to manipulation. I-Theory. II-Implementation. III-Applications," ASME, Transactions, Journal of Dynamic Systems, Measurement, and Control (ISSN 0022-0434), vol. $107,1985$.

[11] H. Kazerooni, P. Houpt, and T. Sheridan, "Robust compliant motion for manipulators: the fundamental concepts of compliant motion (part I);design method (part II)," Robotics and Automation, IEEE Journal of [legacy, pre-1988], vol. 2, no. 2, pp. 83-105, 1986.

[12] R. Murray, Z. Li, and S. Sastry, A mathematical introduction to robotic manipulation. CRC, 1994.

[13] D. Surdilovic, "Contact stability issues in position based impedance control: Theory and experiments," in 1996 IEEE International Conference on Robotics and Automation, 1996. Proceedings., vol. 2, 1996.

[14] Z. Xue, A. Kasper, J. M. Zoellner, and R. Dillmann, "An automatic grasp planning system for service robots," in 14th International Conference on Advanced Robotics (ICAR), 22-26 Jun. 2009.

[15] A. Miller and P. Allen, "Graspit! a versatile simulator for robotic grasping," IEEE Robotics \& Automation Magazine, vol. 11, no. 4, pp. 110-122, 2004. 